\title{
Quantification of hole-trap concentration in degraded polymer light-emitting diodes using impedance spectroscopy
}

Cite as: Appl. Phys. Lett. 114, 163301 (2019); https://doi.org/10.1063/1.5083036

Submitted: 27 November 2018 . Accepted: 06 April 2019 . Published Online: 25 April 2019

Quan Niu, Gert-Jan A. H. Wetzelaer, Paul W. M. Blom (D), and N. Irina Crăciun
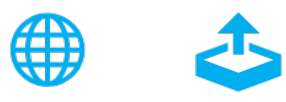

\section{ARTICLES YOU MAY BE INTERESTED IN}

Determination of bimolecular recombination constants in organic double-injection devices using impedance spectroscopy

Applied Physics Letters 114, 123301 (2019); https://doi.org/10.1063/1.5066605

Morphology control of $\mathrm{CsPbBr}_{3}$ films by a surface active Lewis base for bright all-inorganic perovskite light-emitting diodes

Applied Physics Letters 114, 163302 (2019); https://doi.org/10.1063/1.5094041

Efficiency loss processes in hyperfluorescent OLEDs: A kinetic Monte Carlo study

Applied Physics Letters 114, 073301 (2019); https://doi.org/10.1063/1.5079642

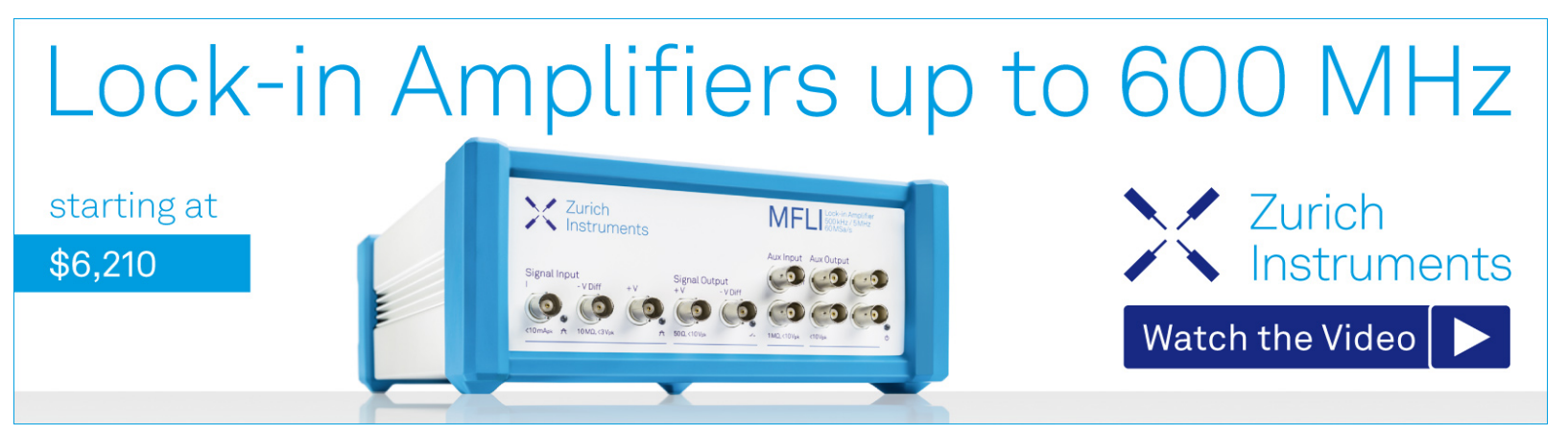




\title{
Quantification of hole-trap concentration in degraded polymer light-emitting diodes using impedance spectroscopy
}

Cite as: Appl. Phys. Lett. 114, 163301 (2019); doi: 10.1063/1.5083036

Submitted: 27 November 2018 - Accepted: 6 April 2019 •

Published Online: 25 April 2019

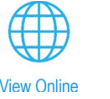

Quan Niu, Gert-Jan A. H. Wetzelaer, Paul W. M. Blom, (D and N. Irina Crăciun ${ }^{a)}$

\author{
AFFILIATIONS \\ Max Planck Institute for Polymer Research, Ackermannweg 10, 55128 Mainz, Germany \\ a) Author to whom correspondence should be addressed: craciun@mpip-mainz.mpg.de
}

\begin{abstract}
The degradation of polymer light-emitting diodes (PLEDs) under current stress is governed by the formation of hole traps. The presence of traps is reflected in the low-frequency response of PLEDs by a negative contribution to the capacitance that originates from trap-assisted recombination. Since the relaxation time scales with the (inverse) concentration of traps, impedance spectroscopy measurements allow for a quantitative determination of the amount of traps formed during degradation. We demonstrate that the obtained hole trap concentration is in agreement with the amount found by numerically modeling the increase in the PLED driving voltage. Impedance spectroscopy measurements are therefore useful as an in-situ characterization tool during PLED degradation, providing information on trap formation without numerical device modeling.
\end{abstract}

Published under license by AIP Publishing. https://doi.org/10.1063/1.5083036

Polymer light-emitting diodes (PLEDs) are attractive candidates for large-area applications as lighting and displays due to potential low-cost fabrication by coating and printing techniques. A bottleneck for their further development has been their relatively low stability and lifetime. Typically, when stressed under a constant current, PLEDs exhibit a continuous increase in operation voltage and a decrease in luminance. ${ }^{1}$ As a possible degradation mechanism, photo-oxidation of the polymer backbone has been proposed. ${ }^{2-8}$ Such photo-oxidation could be initiated or enhanced by the presence of triplet excitons. Triplet excitons not only have a long lifetime but could also activate triplet oxygen to singlet oxygen, which is reactive and could attack the conjugated backbone of the polymer. The electrical characteristics of degraded PLEDs were recently analyzed using a numerical PLED device model. ${ }^{9}$ It was found that the decrease in the PLED current and light-output of degraded PLEDs could be simultaneously explained by the formation of hole traps. Having identified hole trap formation as the main mechanism for degradation enabled us to further investigate the dynamics of trap formation under current stress. ${ }^{10}$ For this purpose, numerical simulations were performed that linked the PLED voltage increase during stress directly to the amount of hole traps. It was found that after a short linear increase in the amount of hole traps with stress time, the hole trap formation is characterized by a square root dependence on time. Furthermore, from the voltage increase, the luminance decay of the PLED could be predicted. The luminance decay under current stress is linked to the hole trap formation via nonradiative recombination of trapped holes with free electrons. The dynamics of the hole trap formation reveals that the degradation is governed by the interactions between excitons and free charge carriers. ${ }^{10}$

For such an in-depth analysis of the electrical characteristics of degraded PLEDs, knowledge of the transport parameters of the pristine polymer is required. Electron and hole mobilities including its field and density dependence, electron trapping, and recombination mechanism are needed to obtain quantitative results using a numerical device model. ${ }^{11}$ For screening of PLED materials, a more direct determination of the amount of traps formed during stress would be convenient. Impedance spectroscopy (IS) is a powerful technique to study charge transport and recombination in PLEDs. At low frequencies, a negative contribution to the differential capacitance $C$ has been observed. ${ }^{12-18}$ Several explanations were proposed, including bimolecular recombination, ${ }^{12}$ electron injection through interfacial states, ${ }^{13}$ accumulation of charges at an organic/organic interface, ${ }^{14}$ trapassisted monomolecular recombination, ${ }^{15,16}$ self-heating, ${ }^{17}$ and energetic disorder. ${ }^{18}$ In a recent study, it has been proven that the negative contribution to the capacitance in pristine PLEDs originates from trap-assisted recombination. ${ }^{19}$ First, it was demonstrated that the 
observed relaxation time is nearly equal to the inverse rate for trapassisted recombination of trapped electrons with free holes. Subsequently, by increasing the amount of electron traps upon addition of a known amount of fullerene molecules, the negative contribution to the capacitance was further amplified and the decrease in the relaxation time was quantitatively in excellent agreement with trapassisted recombination. As final and definite proof, it was shown that when trap-assisted recombination is suppressed by using trapdilution, the negative capacitance effect disappears. The direct correlation between the negative capacitance effect and trap-assisted recombination makes IS a potential tool for investigation of trap formation in PLEDs under current stress. In an earlier study on small molecule based organic LEDs (OLED), IS has been applied to monitor charge injection during degradation. ${ }^{20}$ It was found that under current stress, positive charges accumulated at the interface between the hole- and electron transport layers. The amount of accumulated positive charge was correlated with the rate of degradation. For PLEDs, currentvoltage and impedance spectroscopy measurements have been performed on pristine and degraded PLEDs. ${ }^{10}$ It was demonstrated that the amount of hole traps obtained from the negative capacitance effect is in agreement with the amount of hole traps obtained by numerically modeling the voltage increase during PLED current stress. In the present study, a quantitative analysis of the negative capacitance effect is provided. It is demonstrated that the reduction of the relaxation time for the negative capacitance effect upon degradation is consistent with the formation of hole traps.

Recombination of electrons and holes results in a recombination current $j_{r}$. Its contribution to the capacitance can be related to a time domain response on a voltage step $\Delta V$. It was demonstrated by Ershov et al. ${ }^{21}$ that a positive derivative of the transient recombination current $d j_{r} / d t$ will result in a negative contribution to the capacitance. For a single exponential transient, given by $j_{r}(t)=-j_{0} \exp \left(-t / \tau_{r}\right)$, with $j_{0}$ being the prefactor of the recombination current $j_{r}$, the capacitance is given by

$$
C(\omega)=C_{0}-\frac{\alpha \tau_{r}}{1+\omega^{2} \tau_{r}^{2}},
$$

with $C_{0}$ being the geometrical capacitance $\varepsilon_{0} \varepsilon_{r} A / d, \omega$ the angular frequency, $\alpha$ a proportionality factor that scales with $j_{0}$, and $\tau_{r}$ the relaxation time. ${ }^{21}$ The proportionality factor $\alpha$ is voltage dependent and is proportional to the PLED current. ${ }^{19}$ The near-exponential dependence of $\alpha(V)$ is therefore the result of the density- and field dependent charge carrier mobility in the space-charge limited PLED. The relaxation time $\tau_{r}$ is characteristic of the (inverse) rate of trapped assistedrecombination. In a pristine PLED, where the hole transport is trapfree, ${ }^{11}$ the rate for trap-assisted recombination of free holes with trapped electrons can be approximated by

$$
R_{S R H} \approx C_{p} N_{t} p / 2
$$

with $C_{p}$ representing the hole capture coefficient, $N_{t}$ the total amount of electron traps, and $p$ the density of free holes. In this approximation, it is assumed that $C_{n}=C_{p}$ and $n \approx p$.

This results in a voltage independent relaxation time $\tau_{r}$ given by

$$
\tau_{r}=\frac{2}{N_{t} C_{p}} .
$$

Upon aging, hole traps are formed in a PLED. ${ }^{10,11}$ An increase in the amount of hole traps is then expected to amplify the negative capacitance effect due to the increased trap-assisted recombination of free electrons with trapped holes.

In this study, PLEDs based on a poly(p-phenylene vinylene) (PPV) based copolymer, known as "super yellow" (SY-PPV, Merck $\mathrm{AG}){ }^{22}$ were fabricated and aged under a constant current density of $10 \mathrm{~mA} / \mathrm{cm}^{2}$ for $3 \mathrm{~h}, 17 \mathrm{~h}$, and $56 \mathrm{~h}$. The SY-PPV was dissolved in toluene and spin-coated onto a glass/ITO/poly(3,4-ethylenedioxythiophene):poly(styrenesulfonic acid) (PEDOT:PSS) substrate. The device architecture of the PLED is ITO/PEDOT/SY-PPV $(100 \mathrm{~nm}) / \mathrm{Ba}$ $(5 \mathrm{~nm}) / \mathrm{Al}(100 \mathrm{~nm})$. Impedance data were taken using an Agilent 4284a RCL meter, with the DC bias swept from $-2 \mathrm{~V}$ to $5 \mathrm{~V}$, superposed by an AC bias of $100 \mathrm{mV}$. All the devices were fabricated and measured under an inert $\mathrm{N}_{2}$ atmosphere.

As shown in Fig. 1, at negative bias, the PLED is fully depleted and the measured capacitance equals $C_{0}$, the geometric capacitance of the device. When the applied voltage exceeds the built-in voltage $\left(V_{b i}\right)$ of the PLED, the negative contribution to the capacitance sets in and the capacitance decreases with voltage until it reaches a minimum. At higher voltages, the amount of injected charges strongly increases and charge accumulation regions close to the electrodes are formed, leading again to an increase in the capacitance (the electrodes "grow" into the active polymer layer). In this regime, Eq. (1) is no longer valid. We observe that the negative capacitance effect gets more pronounced with aging and shifts to higher voltages, which indicates that upon aging, the trap-assisted recombination is enhanced. In Fig. 2, the differential capacitance $\mathrm{C}$ vs frequency at various bias voltages for the pristine (a) SY-PPV PLED and PLED stressed at a constant current of $10 \mathrm{~mA} / \mathrm{cm}^{2}$

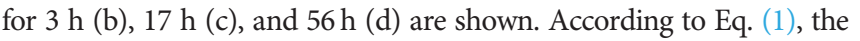
negative contribution to the capacitance can be described by $\alpha(V)$, being proportional to the PLED current, and a constant relaxation time $\tau_{r}$. For the pristine SY-PPV PLED, a $\tau_{r}$ value of $2.7 \mathrm{~ms}$ was found, which decreased upon degradation to $2.0 \mathrm{~ms}(3 \mathrm{~h}), 1.4 \mathrm{~ms}(17 \mathrm{~h})$, and $0.95 \mathrm{~ms}(56 \mathrm{~h})$. We note that a voltage independent $\tau_{r}$ is characteristic of trap-assisted recombination since for bimolecular recombination, $\tau_{r}$ should decrease with voltage due to the increase in carrier density.

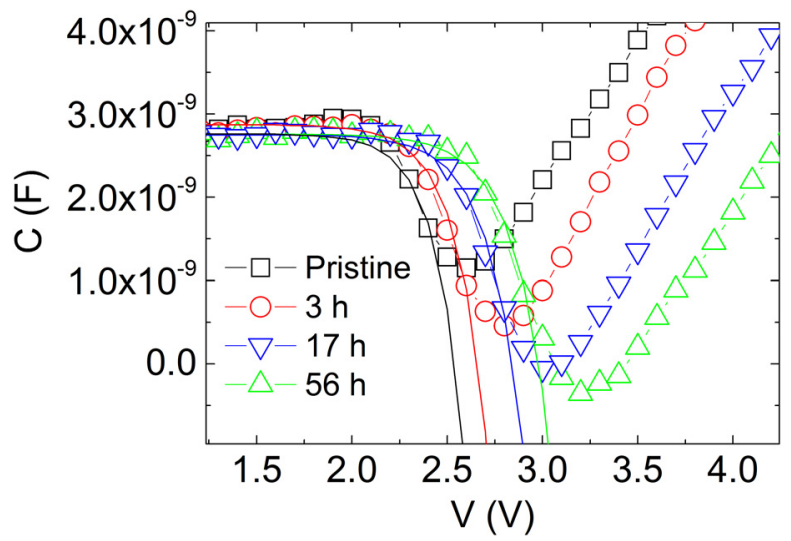

FIG. 1. Measured $C-V$ characteristics (symbols) $(f=100 \mathrm{~Hz})$ of a pristine SY-PPV PLED and PLEDs stressed at a constant current of $10 \mathrm{~mA} / \mathrm{cm}^{2}$ for different aging periods. The PLED active layer thickness amounts to $100 \mathrm{~nm}$. The solid lines are calculated using Eq. (1). The experimental data were taken from Ref. 10, Fig. S2(a). 

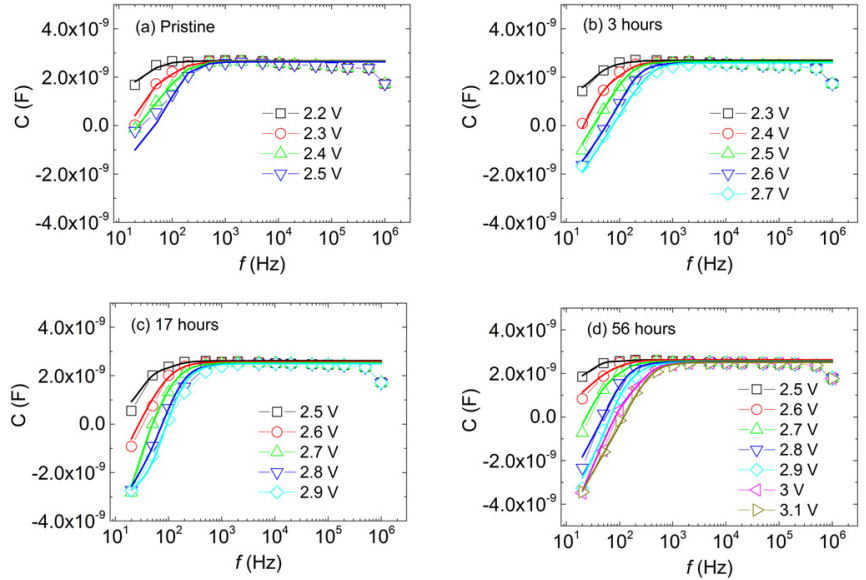

FIG. 2. Differential capacitance $C$ vs frequency at various bias voltages for (a) pristine SY-PPV PLED and (b) PLED stressed at a constant current of $10 \mathrm{~mA} / \mathrm{cm}^{2}$ for $3 \mathrm{~h}$, (c) $17 \mathrm{~h}$, and (d) $56 \mathrm{~h}$. The empty symbols represent the experimental data for an active layer thickness of $100 \mathrm{~nm}$, while the solid lines are a fit to Eq. (1).

The corresponding values of $\alpha(V)$ are shown in Fig. 3 (symbols), together with the normalized measured PLED currents (lines). As reported before for the pristine PLED, ${ }^{19}$ it is demonstrated here that also for the aged devices, $\alpha(V)$ is proportional to the PLED current. The obtained $\alpha(V)$ values together with the above-mentioned values of $\tau_{r}$ are then used in combination with Eq. (1) (Fig. 1, lines) to reconstruct the measured $C$ - $V$ characteristics $(f=100 \mathrm{~Hz})$ of PLEDs aged at a constant current of $10 \mathrm{~mA} / \mathrm{cm}^{2}$ for different periods (Fig. 1, symbols). The calculated $C-V$ characteristics consistently describe the negative capacitance effect upon aging.

The formation of hole traps upon aging leads to additional trapassisted recombination of free electrons with trapped holes. Since in PPV derivatives, the mobilities of free electrons and holes are equal, ${ }^{11}$ the capture coefficients $C_{n}$ and $C_{p}$ are also equal. As a result, the relaxation time for recombination of free holes with trapped electrons [Eq. (3)] has a similar prefactor as the recombination of free electrons with trapped holes so that both contributions can be simply added. The

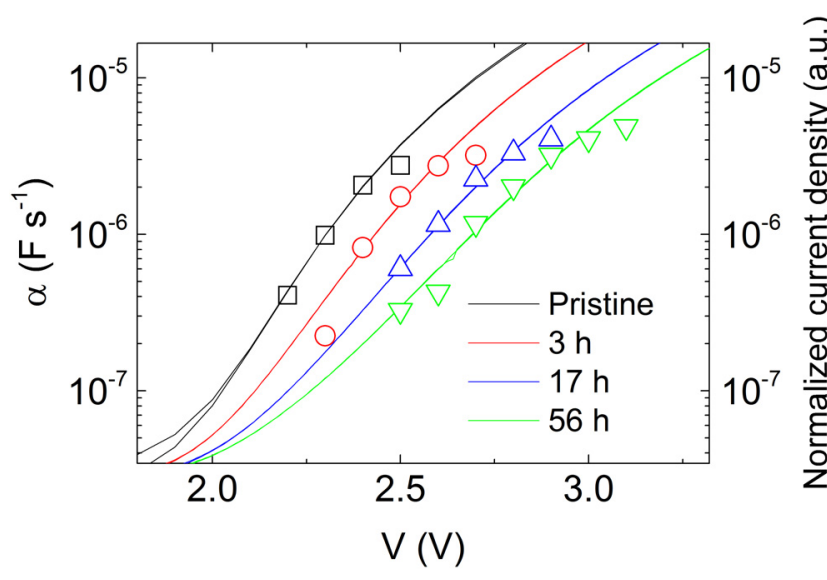

FIG. 3. Voltage dependence of proportionality factor $\alpha(V)$ (symbols) and normalized experimental current densities of pristine and aged SY-PPV PLEDs (solid lines). relaxation time for aged PLEDs is then governed by the total amount of traps $N_{t o t}=N_{t 0}+P_{t}$, with $N_{t 0}=1 \times 10^{23} \mathrm{~m}^{-3}$ being the density of electron traps in a pristine device and $P_{t}$ the amount of hole traps generated during degradation. The total amount of traps $N_{t o t}$ can now be directly obtained from the obtained relaxation times of the aged devices using

$$
\mathrm{N}_{\mathrm{tot}}=\tau_{\mathrm{r}}^{-1}\left(\tau_{0} \mathrm{~N}_{\mathrm{t} 0}\right)
$$

with $\tau_{0}=2.7 \mathrm{~ms}$ and $N_{t 0}=1 \times 10^{23} \mathrm{~m}^{-3}$. In Fig. 4, the total amount of traps $N_{t o t}$ is shown as a function of the obtained $\tau_{r}{ }^{-1}$ for the pristine and aged PLEDs (black squares). The amount of hole traps upon aging is then obtained from $P_{t}=N_{t o t}-N_{t 0}$ (red dots). We note that for a quantitative determination of the hole trap concentration upon degradation, knowledge of $N_{t 0}$ is required, which can be independently determined from charge transport measurements using hole-only and electron-only devices in combination with pristine PLEDs.

In order to maintain a constant current for PLEDs under stress, the generated hole traps, leading to immobile holes in the device, will cause the voltage to rise. From the recorded voltages, the density of hole traps $P_{t}(t)$ as a function of aging time can be calculated using a numerical PLED device model. ${ }^{10}$ In Fig. 5, the numerically calculated amount of hole traps $P_{t}(t)$ as a function of aging time is shown for a SY-PPV PLED stressed at $10 \mathrm{~mA} / \mathrm{cm}^{2}$. As reported earlier, the formation of hole traps is governed by linear growth at short aging times and a square-root dependence at longer times. ${ }^{10}$ Also included in Fig. 5 are the hole trap concentrations obtained from the relaxation times of the negative contribution to the capacitance, which are shown in Fig. 4 (circles).

We observe that the obtained hole trap concentrations from both methods agree very well. This demonstrates that recombination of free electrons and trapped holes after degradation indeed contributes in an equal way to the negative capacitance as the recombination of free holes with trapped electrons in pristine PLEDs. The quantitative agreement also demonstrates that impedance spectroscopy can be used as a tool to study degradation in organic devices by enabling a direct determination of the trap densities from the observed relaxation times. The only other additional measurement needed is the $J-V$ characteristic of

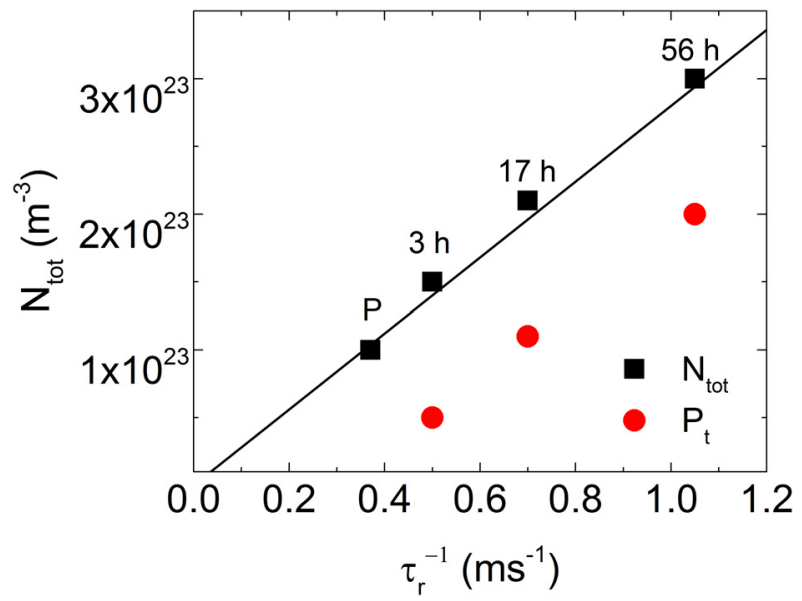

FIG. 4. Total density of traps (squares) and hole traps (circles) for a pristine (P) SYPPV (no hole traps) and after aging for 3,17 , and $56 \mathrm{~h}$ at $10 \mathrm{~mA} / \mathrm{cm}^{2}$. 


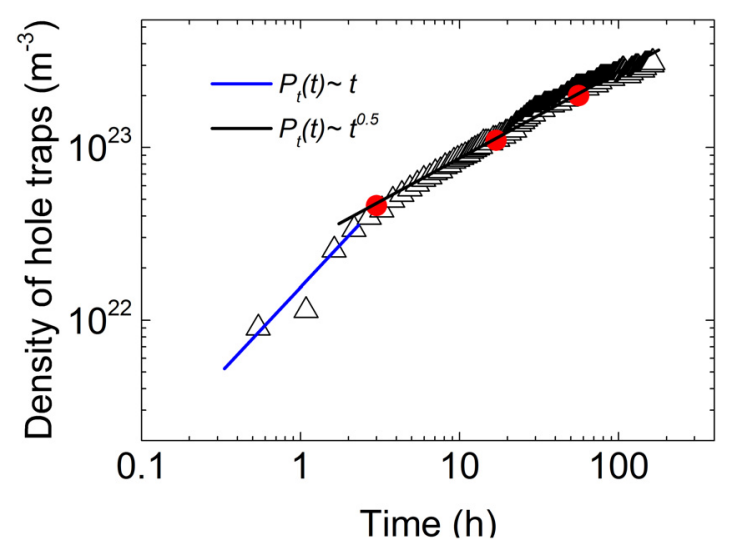

FIG. 5. Density of hole traps formed during degradation at $10 \mathrm{~mA} / \mathrm{cm}^{2}$ calculated from the voltage increase (open symbols) and the hole trap concentration obtained from capacitance measurements (closed symbols) of a SY-PPV based PLED. The experimental data were taken from Ref. 10, Fig. S2(b).

the degraded PLEDs to incorporate the decrease in the prefactor $\alpha(V)$ upon degradation. For multilayer OLEDs, such a straightforward analysis of IS data is more complicated due to the presence of many interfaces and guest-host layers where charge trapping can occur, which may all affect the frequency response.

In conclusion, we have demonstrated that the enhanced negative capacitance effect in degraded PLEDs, in which hole traps are generated by current stress, can be attributed to trap-assisted recombination of free electrons with trapped holes. From the observed relaxation times, the amount of generated hole traps can be quantitatively determined for various aging times. The obtained trap concentrations are consistent with the hole traps determined from the PLED driving voltage by numerical simulations. As a result, impedance spectroscopy can be used as a tool for monitoring trap concentrations during degradation of organic devices.

\section{REFERENCES}

${ }^{1}$ I. D. Parker, Y. Cao, and C. Y. Yang, J. Appl. Phys. 85, 2441 (1999).

${ }^{2}$ J. R. Sheats and D. B. Roitman, Synth. Met. 95, 79 (1998).

${ }^{3}$ M. Yan, L. Rothberg, F. Papadimitrakopoulos, M. Galvin, and T. Miller, Phys. Rev. Lett. 73, 744 (1994).

${ }^{4}$ R. D. Scurlock, B. Wang, P. R. Ogilby, J. R. Sheats, and R. L. Clough, J. Am. Chem. Soc. 117, 10194 (1995).

${ }^{5}$ B. H. Cumpston and K. F. Jensen, Trends Polym. Sci. 4, 151 (1996).

${ }^{6}$ J. C. Scott, J. Kaufman, P. Brock, R. DiPietro, J. Salem, and J. Goitia, J. Appl. Phys. 79, 2745 (1996).

${ }^{7}$ B. H. Cumpston, I. D. Parker, and K. F. Jensen, J. Appl. Phys. 81, 3716 (1997).

${ }^{8}$ E. Staring, A. Berntsen, S. Romme, G. Rikken, and P. Urbach, Philos. Trans. R. Soc. London A 355, 695 (1997).

${ }^{9}$ Q. Niu, G. A. H. Wetzelaer, P. W. M. Blom, and N. I. Crăciun, Adv. Electron. Mater. 2, 1600103 (2016).

${ }^{10}$ Q. Niu, R. Rohloff, G. A. H. Wetzelaer, P. W. M. Blom, and N. I. Crăciun, Nat. Mater. 17, 557-562 (2018).

${ }^{11}$ M. Kuik, G. A. H. Wetzelaer, H. T. Nicolai, N. I. Craciun, D. M. De Leeuw, and P. W. M. Blom, Adv. Mater. 26, 512 (2014).

${ }^{12}$ H. H. P. Gommans, M. Kemerink, and R. A. J. Janssen, Phys. Rev. B 72, 235204 (2005).

${ }^{13}$ J. Bisquert, G. Garcia-Belmonte, A. Pitarch, and H. J. Bolink, Chem. Phys. Lett. 422, 184 (2006).

${ }^{14}$ L. S. C. Pingree, M. T. Russell, T. J. Marks, and M. C. Hersam, J. Appl. Phys. 100, 044502 (2006).

${ }^{15}$ E. Ehrenfreud, C. Lungenschmied, G. Dennler, H. Neugebauer, and N. S. Sariciftci, Appl. Phys. Lett. 91, 012112 (2007).

${ }^{16}$ T. K. Djidjou, T. Basel, and A. Rogachev, J. Appl. Phys. 112, 024511 (2012).

${ }^{17}$ E. Knapp and B. Ruhstaller, J. Appl. Phys. 117, 135501 (2015).

${ }^{18}$ W. C. Germs, S. L. M. van Mensfoort, R. J. de Vries, and R. Coehoorn, J. Appl. Phys. 111, 074506 (2012).

${ }^{19}$ Q. Niu, N. I. Crăciun, G. A. H. Wetzelaer, and P. W. M. Blom, Phys. Rev. Lett. 120, 116602 (2018).

${ }^{20}$ S. Nowy, W. Ren, A. Elschner, W. Lövenich, and W. Brütting, J. Appl. Phys. 107, 054501 (2010).

${ }^{21}$ M. Ershov, H. C. Liu, L. Li, M. Buchanan, Z. R. Wasilewski, and A. K. Jonscher, IEEE Trans. Electron Devices ED-45, 2196 (1998).

${ }^{22}$ H. Spreitzer, H. Becker, E. Kluge, W. Kreuder, H. Schenk, R. Demandt, and H. Schoo, Adv. Mater. 10, 1340 (1998). 